\title{
Anaphylactic reaction in patient allergic to mango
}

\author{
Natalia Ukleja-Sokołowska ${ }^{1 *}$, Ewa Gawrońska-Ukleja' ${ }^{1}$ Kinga Lis' ${ }^{1}$, Magdalena Żbikowska-Gotz' , \\ Łukasz Sokołowski ${ }^{2}$ and Zbigniew Bartuzi ${ }^{1}$
}

\begin{abstract}
Background: An allergy to mango is extremely rare. The antigenic composition of the fruit is not fully known. Profilin from mango has a structure similar to birch tree profiling: it is responsible for cross-reactions between mango and pear, apple, and peach. A panallergen with a structure similar to mugwort defensin (Art v 1) which cross-reacts with celery, carrot, peanuts, pepper, aniseed, and caraway has been previously described.
\end{abstract}

Case study: A female patient, 30 years old, was admitted in February 2017 because of recurrent allergic reactions following consumption of various foods. The most severe allergic reaction in the patient's life occurred after eating a mango fruit. Within several minutes the patient developed a generalised urticaria, followed by facial oedema, strong stomach pain and watery diarrhoea. The diagnostics involved skin tests with a set of inhalatory and food allergens, including native skin tests. The patient also experienced symptoms of recurrent, generalized urticaria in connection with consumption of various types of food, especially complex dishes containing many different ingredients. Additionally, an interview revealed that the patient was experiencing symptoms of the oral allergy syndrome after ingesting various fruit and vegetables, especially during late summer and fall. Diagnostics was extended by determining the levels of IgE specific for allergen components, using the ImmunoCap ISAC method. In order to confirm the occurence of a cross-reaction between mugwort and mango allergens, we performed the inhibition test of IgE specific for mugwort using a mango allergen extract and ImmunoCap matrix.

Results: Skin prick tests (SPT) were positive for allergens of grass $7 \mathrm{~mm}$; weeds $8 \mathrm{~mm}$; cat's fur $5 \mathrm{~mm}$; mugwort $6 \mathrm{~mm}$. SPT were also positive for mango. The level of specific lgE was increased for allergens of mugwort, grass, celery, pepper, carrot, mango, banana, peach, and apple. The ImmunoCap ISAC test demonstrated a high level of specific IgE rPhl p 1 (timothy grass) and Art $v 1$ (mugwort). We also performed the IgE inhibition test using both mango extract and ImmunoCap matrix and confirmed a cross-reaction with Art $\vee 1$ in the pathogenesis of symptoms observed in the patient.

Conclusions: An anaphylactic reaction to consumed mango, resulting from cross-allergy with mugwort Art $v 1$ was diagnosed in the patient. Acute urticarial in this case is a manifestation of IgE-mediated food allergy. During in vitro diagnostic procedures we found an elevated concentration of IgE specific to several food allergens (including celery, peppers, carrot, banana, peach, apple, shrimp). The elimination diet removing allergens the patient was allergic to was recommended. Considering the anaphylactic reaction the patient was instructed to carry a rescue set composed of an adrenaline autosyringe, steroids, and antihistamines.

Keywords: Mango, Allergy, Mugwort, Inhibition test, Art v 1

\footnotetext{
*Correspondence: ukleja@10g.pl

${ }^{1}$ Department and Clinic of Allergology, Clinical Immunology and Internal

Diseases, Ludwik Rydygier Collegium Medicum in Bydgoszcz, NCU,

Bydgoszcz, Poland

Full list of author information is available at the end of the article
} 


\section{Background}

Mango (Latin: Mangifera indica), called the "king of fruit", is one of the most commonly grown exotic fruits. It originally comes from South Asia. The plant belongs to the Anacardiaceae family. Other representatives of the same family are pistachios and cashew nuts [1].

Mango has been cultivated for approximately 6000 years. In India the fruit is referred to as the "fruit of the gods". It is the most important fruit in India. Mango constitutes half of the whole fruit production in India.

Indian mango is a popular edible fruit. The fruit is a drupe, with a large pit. Mango contains a glycoside called mangiferin which possesses anti-viral, anti-microbial, anti-atherosclerotic, and anti-diabetic properties. The fruit is also a rich source of beta carotene and vitamin $\mathrm{C}$ [2].

An allergy to mango is extremely rare. Hypersensitivity to mango allergens may be immediate or delayed. In 2011 Sareen et al. performed a meta-analysis of cases of allergic reactions to mango available in the Medline/ Pubmed database. They found 17 papers concerned with a total of 22 patients. Ten of these patients had immediate reactions, and the rest had delayed reactions. The most common symptoms included: wheezing, urticaria, angioedema, and anaphylaxis [3, 4].

The antigenic composition of the fruit is not fully known. 334 different proteins were found in mango peel, and 2855 in the fruit flesh. Some of these proteins may possess immunizing properties [5].

The following mango allergens have been identified to date:

- Man i 1-the major allergen, molecular weight of $40 \mathrm{kDa}$ and unknown function;

- Man i 2-the major allergen, molecular weight of $30 \mathrm{kDa}$ and unknown function;

Little is known about the biological role of those allergens. In 2017 Tsai et al. [6] described the sequence of Man i 1 and its expression protocol and subsequent replication in Escherichia coli cell during mitosis.

- Man i 3-a minor allergen, a cross-reacting profilin [7] - a panallergen with a structure similar to mugwort defensin (Art v 1) which cross-reacts with celery, carrot, peanuts, pepper, aniseed, and caraway was also described in this study. It was discovered that allergens from mango may cross-react with latex allergens, through the phenomenon known as the latex-fruit syndrome $[4,7]$.

Mugwort (Latin Artemisia vulgaris) is the most important representative of the Asteraceae family. It is one of the main causes of allergic reactions in Europe. It is estimated that approximately $95 \%$ of patients allergic to mugwort have IgE against Art v 1, the main allergen, a glycoprotein with a defensin-like domain $[8,9]$.

\section{The case study}

A female patient, 30 years old, was admitted in February 2017 to the Department and Clinic of Allergology, Clinical Immunology and Internal Diseases because of recurrent allergic reactions following consumption of various foods.

These reactions were largely dermal, in the form of urticaria on the whole body. Symptoms of recurrent, generalized urticaria were experienced after ingesting various especially complex dishes containing many different ingredients. The patient associated those symptoms with consumption of peppers, clementines, buckwheat honey, and red wine.

The most severe allergic reaction in the patient's life occurred after eating a mango fruit. Within several minutes the patient developed a generalised urticaria, followed by facial oedema, strong stomach pain, and watery diarrhoea. Paramedics were called. The patient was administered adrenaline, steroids, and antihistamines.

A detailed medical history revealed that for the previous 6 years the patient had symptoms of seasonal rhinitis (lacrimation, itching eyelids, and watery nasal discharge).

The patient at the time was also being treated with levothyroxine for hypothyroidism. The patient's family history is ridden with allergic diseases: the patient's mother is allergic to inhalatory allergens, and her father to birch and cat.

In this complex case diagnosis required a wide range of diagnostic methods. As a part of the diagnostics performed in the Department and Clinic of Allergology, Clinical Immunology and Internal Diseases, the patient had skin prick tests with a set of inhalatory allergens (Dermatophagoides farinae, Dermatophagoides pteronyssinus, grass/cereals, weeds, Aspergillus fumigatus, Cladosporium herbarum, cat's fur, poplar, hazel, alder, birch, mugwort, ribwort plantain) and food allergens (chicken egg, cow milk, tomato, carp, banana, rye flour, wheat flour, peanuts, hazelnut, pork, chicken meat, orange) from Allergopharma-Nexter Sp. $\mathrm{z}$ o.o. (Ltd.), as well as native skin tests with fresh foods (buckwheat honey, peppers, mango, clementine, carrot, celery, peanuts, banana, mustard, turmeric, caraway, red wine).

Levels of IgE specific for selected allergens (mugwort, wormwood, early grass mix, late grass mix, celery, peppers, carrot, mango, banana, peach, apple, peanuts, hazelnuts, Dermatophagoides pteronyssinus, 
Dermatophagoides farinae, early trees mix, late trees mix, birch, Cladosporium herbarum, Aspergillus fumigatus, Alternaria tenius, cow milk protein, seasoning mix, mustard) were determined with the ImmunoCap (ThermoFisher Scientific, Uppsala, Sweden) system, using the Phadia100 equipment according to the manufacturer's instructions (ImmunoCAP specifi IgE, fluoroenzymeimmunoassay, 52-5291-EN/05).

The diagnostics were expanded by a determination of the level of IgE specific for allergen components, using the ImmunoCap ISAC method.

Each of these tests have different specificity and sensitivity and, no less importantly, availability and cost. Skin prick tests are considered a fast, inexpensive method with immediate results. Unfortunately, their detection rate in food allergy is limited. In a recent article by Griffiths et al. [10] the detection rates for SPTs (53\%) and ISAC (59\%) were similar, with a higher detection rate for ImmunoCAP testing (66\%). In patients with nut allergies, tests for sensitisation to nuts scored similarly, but with a greater sensitivity (71\%) for ImmunoCAP tests than SPT (53\%) or ISAC (65\%). Therefore we extended the diagnosis in this case to in vitro determination of the concentration of allergen specific IgE. The gold standard in diagnosis of food allergy is still the double blind placebo controlled food challenge. In this case the patient did not consent to provocation challenges.

Skin prick tests were positive for the following allergen extracts (wheal average diameter in millimetres): grass $7 \mathrm{~mm}$; weeds: $8 \mathrm{~mm}$; cat's fur: $5 \mathrm{~mm}$; mugwort: $6 \mathrm{~mm}$; ribwort plantain: $4 \mathrm{~mm}$; celery: $2 \mathrm{~mm}$; clementine: $2 \mathrm{~mm}$ (histamine $4 \mathrm{~mm}$, negative control $0 \mathrm{~mm}$, a result was interpreted as positive in case of a wheal average diameter $\geq 3 \mathrm{~mm}$ ). No skin reaction was observed for other tested allergen extracts. The total IgE level was $406.53 \mathrm{kU} / \mathrm{L}$.

Results of native tests were positive for mango $5 \mathrm{~mm}$, celery $3 \mathrm{~mm}$.

Results for specific IgE levels tested using the ImmunoCap method are presented in Table 1. Elevated levels of IgE (above $0.35 \mathrm{kU} / \mathrm{L}$ ) were found against mugwort, wormwood, early grass mix, late grass mix, celery, peppers, carrot, mango, banana, peach, apple, peanuts.

After the patient was discharged from the hospital we received results of the ImmunoCap ISAC tests (Table 2). A high level of IgE specific for Art $\mathrm{v} 1$ (defensin) from mugwort and Phl p 1 from timothy was particularly noteworthy. It is worth to emphasize that there were no elevated levels of IgE specific to components of food allergens available in ImmunoCap ISAC. It is possible that symptoms of OAS in this patient resulted from cross reactivity with timothy
Table 1 The list of results for specific IgE levels tested using the ImmunoCap method

\begin{tabular}{|c|c|c|}
\hline No. & Allergen & IgE level (kU/L) \\
\hline 1 & Mugwort & 144.32 \\
\hline 2 & Wormwood & 82.83 \\
\hline 3 & Early grass mix & 37.14 \\
\hline 4 & Late grass mix & 11.77 \\
\hline 5 & Celery & 3.16 \\
\hline 6 & Peppers & 0.98 \\
\hline 7 & Carrot & 0.96 \\
\hline 8 & Mango & 0.94 \\
\hline 9 & Banana & 0.89 \\
\hline 10 & Peach & 0.87 \\
\hline 11 & Apple & 0.59 \\
\hline 12 & Peanuts & 0.39 \\
\hline 13 & Hazelnuts & $<0.35$ \\
\hline 14 & Dermatophagoides pteronyssinus & $<0.35$ \\
\hline 15 & Dermatophagoides farinae & $<0.35$ \\
\hline 16 & Early trees mix & $<0.35$ \\
\hline 17 & Late trees mix & $<0.35$ \\
\hline 18 & Birch & $<0.35$ \\
\hline 19 & Cladosporium herbarum & $<0.35$ \\
\hline 20 & Aspergillus fumigatus & $<0.35$ \\
\hline 21 & Alternaria tenius & $<0.35$ \\
\hline 22 & Cow milk protein & $<0.35$ \\
\hline 23 & Seasonings mix & $<0.35$ \\
\hline 24 & Mustard & $<0.35$ \\
\hline
\end{tabular}

and mugwort allergens. Symptoms of urticaria may be also result from sensitivity to one of the food allergens mentioned above. The only anaphylactic reaction the in patients life occurred after the consumption of mango, and this hypersensitivity was the one that, we decided, required further diagnosis.

Here it needs to be clearly stated that the ImmunoCap ISAC test did not indicate if in that particular case the patient's allergy to mango was a result of a crossallergy with Art v 1, or an allergy to another molecule, independent from mugwort. ImmunoCap ISAC has no mango allergen components available.

To clarify this, then, the ImmunoCap inhibition test was applied, using the allergen extract from fresh mango fruit. The investigation was based on the SPHIA model (Single Point Highest Inhibition Achievable Assay) described by Bernardi et al. in 2011 [11].

The methodology of this inhibition assay is still considered experimental, although similar inhibition assays were applied previously by several authors. For example, in our research unit a case of a patient allergic to sunflower seed was described, where ImmunoCap ISAC inhibition test was used to prove cross reactivity. 
Table 2 ImmunoCap ISAC test results. No increased levels of allergen-specific IgE were found for other allergen components included in the ImmunoCap test

\begin{tabular}{llll}
\hline Allergen source & $\begin{array}{l}\text { Allergen } \\
\text { component }\end{array}$ & Allergen type & $\begin{array}{l}\text { IgE level } \\
\text { (ISU-E) }\end{array}$ \\
\hline Bermuda grass & $\mathrm{nCyn} \mathrm{d} \mathrm{1}$ & Grass group 1 & 4.6 \\
Timothy grass & rPhl p 1 & Grass group 1 & 38 \\
Timothy grass & rPhl p 4 & Berberine bridge enzyme & 2.2 \\
Mugwort & nArt v 1 & Defensin & 34 \\
Cat & rFel d 1 & Uteroglobin & 3.3 \\
Anisakis & rAni s 3 & Tropomyosin & 0.5 \\
Cockroach & nBla g 7 & Tropomyosin & 0.7 \\
$\begin{array}{l}\text { D.pteronyssinus } \\
\text { (house dust }\end{array}$ & rDer p 10 & Tropomyosin & 1 \\
mite) & & & \\
Shrimp & nPen m 1 & Tropomyosin & 0.4 \\
\hline
\end{tabular}

In the inhibition test Art v 1, Art v 3, and Jug r 3 were inhibited by the protein present in the sunflower seed allergen extract [12].

To perform the inhibition test a ripe mango fruit was bought in a local grocery offering eco food. The fruit was washed, peeled and the pit was removed to obtain the edible part. The protein-containing pulp extract was obtained using methods described previously [13, 14]. Diced fruit was frozen in liquid nitrogen and blended to smooth pulp. The pulp was then mixed with $1 \mathrm{M}$ $\mathrm{NaCl}$ in the ratio of $2: 1(2: 1, \mathrm{v} / \mathrm{v})$ and left at $2-8{ }^{\circ} \mathrm{C}$ for $24 \mathrm{~h}$. After that time the mixture was centrifuged at room temperature at $12,000 \times g$ for $60 \mathrm{~min}$. The extract was obtained containing $2.35 \mathrm{mg} / \mathrm{dL}$ of protein. In the inhibition test the obtained extract was mixed with the patient's blood serum at the volume ratio of $1: 1$, and incubated at $2-8{ }^{\circ} \mathrm{C}$ for $24 \mathrm{~h}[11,14]$. A blind sample was simultaneously prepared in order to account for the dilution of the serum. For that purpose, the patient's blood serum was mixed with $1 \mathrm{M} \mathrm{NaCl}(1: 1, \mathrm{v} / \mathrm{v})$, and then incubated at $2-8{ }^{\circ} \mathrm{C}$ for $24 \mathrm{~h}$. After that time, the level of IgE specific for mugwort, Art v1, and mango was determined in the prepared material, both inhibited and diluted, and the inhibition ratio was calculated.

In the inhibition test we achieved the following results-the baseline level of mugwort sIgE in the patient's serum was $144.32 \mathrm{kU} / \mathrm{L}$. In the blind sample, after diluting it with $1 \mathrm{M} \mathrm{NaCl} 1: 1$, the concentration of sIgE was $72.16 \mathrm{kU} / \mathrm{L}$. After incubation of the patient's serum with a mango allergen extract the level of mugwort IgE was $48.81 \mathrm{kU} / \mathrm{L}$. It was thus shown that the mango allergen extract inhibited the binding of mugwort IgE by $32.38 \%$

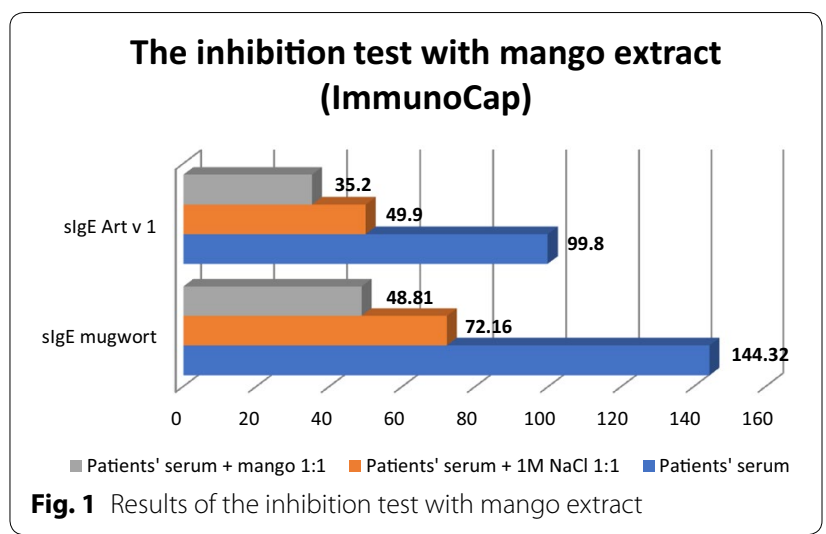

The baseline level of Art v 1 sIgE in the patient's serum was $99.8 \mathrm{kU} / \mathrm{L}$. In the blind sample, after diluting

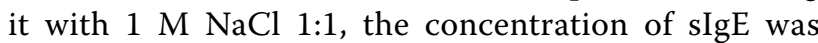
$49.9 \mathrm{kU} / \mathrm{L}$. After incubation of the patient's serum with the mango allergen extract the level of Art v 1 IgE was $35.2 \mathrm{kU} / \mathrm{L}$. It was thus shown that the mango allergen extract inhibited the binding of IgE Art v 1 by $29.5 \%$. Based on the performed diagnostic procedures it was demonstrated that symptoms present in the patient could be a result of a cross-reaction between allergens of mango and Art v 1. Results of the inhibition test are presented in Fig. 1.

Due to the fact that this inhibition test was an experimental method and the previously described protocol was modified to accompany the needs of this specific case, an inhibition test according to a different protocol was performed in order to provide a final confirmation of the cross-allergy. The patient's serum was incubated with the allergen extract from a mango fruit, using the ImmunoCap matrix. The ImmunoCap was pre-washed four times: twice with the wash worksolution ImmunoCap, and twice with the neutral $\mathrm{pH}$ phosphate buffer. $50 \mu \mathrm{L}$ of tested serum was added to the ImmunoCap prepared that way, and incubated for $1 \mathrm{~h}$ at room temperature, and then centrifuged at $1500 \times g$ for $2 \mathrm{~min}$. The obtained antibody-depleted serum, and the native serum were analysed for the presence of the Art v 1 allergen component, using the ImmunoCap method.

The baseline level of Art v 1 sIgE in patients serum was $99.8 \mathrm{kU} / \mathrm{L}$. In this case the patient's serum was not diluted, so negative control was not necessary. After incubation of the patient's serum with ImmunoCap mango matrix the level of Art v $1 \mathrm{IgE}$ was $56.5 \mathrm{kU} / \mathrm{L}$. As a result it was shown that the ImmunoCap mango 


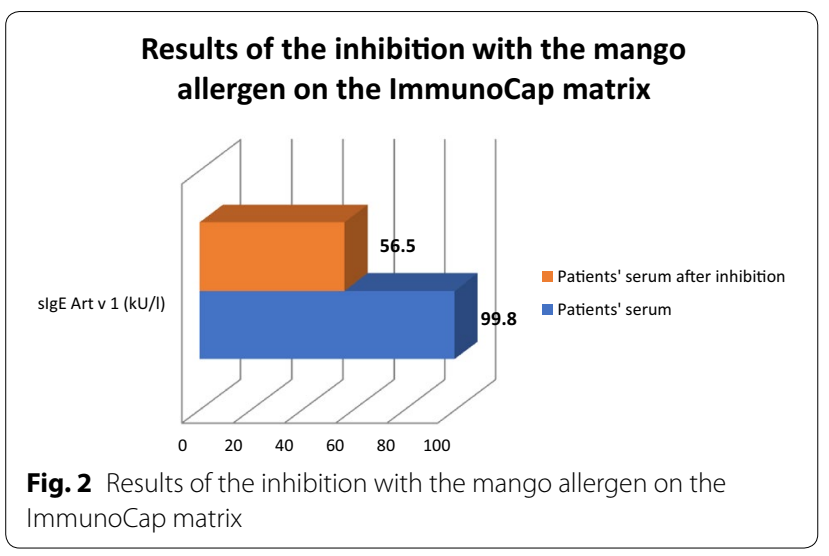

matrix inhibited binding of Art v 1 IgE by 43.4\%. These results are presented in Fig. 2.

\section{Discussion}

An anaphylactic reaction to consumed mango, resulting from cross-allergy with mugwort Art v 1 was diagnosed in the patient. Allergy to various foods was diagnosed, including celery, peppers, carrot, banana, apple, and peanuts. Allergies to grass pollen, timothy, mugwort, and cat's fur were also diagnosed.

The presented case was interesting because of a rarely reported anaphylaxis after the consumption of a mango fruit. Mango allergens have not been characterised precisely [6]. According to reports, there are some mango allergens that may cross-react with birch pollen (profilin) and mugwort pollen (defensin). The diagnostics involved inhibition of IgE specific for the mugwort allergen extract and Art v 1, using the mango allergen extract obtained from fresh mango fruit in our laboratory. It was confirmed that the allergen extract inhibited IgE by $\sim 30 \%$, which confirmed the participation of the crossreaction with Art $\mathrm{v} 1$ in the development of symptoms in the analysed patient.

The inhibition test used for confirmation of the crossallergy as a source of symptoms in a mugwort-allergic patient was described, among others, in 2016: the reported test confirmed a cross-allergy to mugwort Art v 1 and Art $\mathrm{v} 3$ in a patient who developed an anaphylactic reaction following consumption of sunflower seeds [12].

In the present case we used two different protocols for the inhibition assay. Through the first inhibition assay it was shown that the mango allergen extract inhibited the binding of IgE Art v 1 by $29.5 \%$. In the second protocol, the ImmunoCap mango matrix inhibited the binding of Art v 1 IgE by $43.4 \%$. The difference in the inhibition may be with a result of the presence of different antigens in the ImmunoCap Matrix and in the native mango allergen extract, due to different variety of fruit. What is more,
ImmunoCap has a matrix with high bioaccessibility and bioavailability, which improves the binding of serum SIgE to antigens.

Art $\mathrm{v} 1$ is a defensin-like protein. It is relatively stable to proteolytic degradation, which may lead to a higher sensitization rate of Art v 1 compering to other defensinlike proteins [e.g. ragweed (Ambrosia artemisiifolia) Amb a 4 or Santa Maria feverfew (Parthenium hysterophorus) Par h 1] [15]. We might suspect that the mango allergen, cross reactive with Art $\mathrm{v} 1$, is also a stable allergen and for this reason positive results were achieved both during prick by prick test with fresh mango and during immunodiagnostic methods with the mango allergen extract (frozen in liquid nitrogen), and with the ImmunoCap mango allergen extract. We did not perform skin prick tests with a commercial mango allergen extract due to the lack of this allergen in the portfolio of the company from which we acquire extracts for SPT (Allergopharma Nexter).

Reported cases of an allergic reaction to mango are relatevely scarce. In 1942 Kahn described a case of a female patient who "developed some symptoms" after eating a mango fruit. The patient was persuaded to undergo a food challenge test. After eating a mango fruit the patient experienced dyspnoea and wheezing of asthmatic character. Those symptoms disappeared after the administration of adrenaline [16].

In 2009, Silva et al. [17] described a case of a 39-year old female patient who developed an anaphylactic reaction after eating a mango fruit, most probably caused by allergy to the $13 \mathrm{kDa}$ allergen cross-reacting with mugwort allergens, which was confirmed in the immunoblotting inhibition assay.

Hedge et al. [1] described an interesting case of a female patient whose symptoms, occurring after consumption of a mango fruit, were most probably a result of an IgEdependent reaction to a low-molecular-weight protein included in the allergen extract made from a fresh mango fruit. It was observed that similar symptoms occurred in that patient also after ingesting other fruit of the Anacardiaceae family, e.g. a cashew fruit (the edible part of the plant with the cashew nut).

In 2017 Valk et al. [8] published an interesting paper analysing 29 cases of children with a confirmed allergy to cashew nuts. The children underwent an oral challenge test with pistachios and mango fruit. None of the children demonstrated hypersensitivity to mango, which indicated a relative low risk of cross-reactions with cashew nuts.

An interesting report was published in 2015 by Ta et al [18]. The authors described a case of an 8-month old patient who experienced recurrent reactions in the form of vomiting, weakness, decreased muscular strength of extremities, and peripheral cyanosis, several hours after 
consumption of a mango fruit. A detailed diagnostics was performed and an enteritis syndrome caused by a mango protein was diagnosed.

Patients with contact dermatitis involving body parts that had contact with mango pulp were also described $[19,20]$.

Acute urticaria is a common manifestation of IgEmediated food allergy, although food allergy is not the most common cause of acute urticaria and is rarely a cause of chronic urticaria [21]. In the case of the patient described here, during in vitro diagnostic procedures we found an elevated concentration of IgE specific to several food allergens (including celery, peppers, carrot, banana, peach, apple, shrimp). The results may be interpreted alongside-cross reactivity to pollen allergens (for example symptoms of oral allergy syndrome) and co-sensitization to food allergens in pollen allergic patients. Moreover, based on the results alone, it is not possible to distinguish between a clinically relevant allergy and asymptomatic sensitisation. An allergen-specific elimination diet, with careful monitoring of symptoms after eliminating 1-2 specific kinds of food was advised in order to improve the patients symptom's and, at the same time, diagnose the clinically relevant allergy.

According to Boyce et al. [22] elimination of 1 or a few specific foods from the diet may be useful in the diagnosis of food allergy. Although the quality of evidence for this approach is low, it is useful when it is not possible to perform the oral food challenge, the gold standard for diagnosing food allergies. This test is accurate and sensitive, but also presents a greatest risk to the patient. What is more, it is important to remember that prolonged elimination diets that omit multiple foods have been reported to induce severe malnutrition.

Strict elimination of mango, as the cause of anaphylaxis, was advised.

Specific immunotherapy with mugwort allergen extract was recommended to the described patient. Symptomatic treatment of rhinitis was also recommended during pollination seasons. Considering the anaphylactic reaction the patient was also instructed to carry a rescue set composed of an adrenaline autosyringe, steroids, and antihistamines.

On a follow-up visit a detailed interview revealed that the patient did not experience further episodes of anaphylaxis. She strictly avoids mango fruit, and also eliminated celery from her diet. The patient felt that the condition of her skin improved and the episodes of acute urticaria appear not as often and with less intensity. Further observation of symptoms was recommended.

\section{Summary}

The presented case is interesting, because it is one of a few cases of anaphylactic reactions associated with an allergy to a mango fruit. We would like to draw attention to this rare allergen, often discarded during allergy diagnosis, especially due to the lack of commercially available allergen extracts for skin prick testing.

Our particular diagnostic approach is also worth highlighting, because it enabled us to confirm a crossallergy with mugwort Art $\mathrm{v} 1$ as a potential source of anaphylactic symptoms in the patient. Inhibition testing is a relatively simple tool that allows to distinguish between cross reactivity and co-reactivity.

Patients with inhalatory allergies often have typical symptoms of OAS. There is a group of patients that can experience systemic symptoms as well. In the described case cross reactivity resulted in anaphylaxis. Moreover, the patient had several episodes of generalised urticaria which require further observation. The fact, that patient had elevated IgE to several inhalatory and food allergens makes this case more complicated. In real life conditions many patients suffer simultaneously from allergies of different orgin (for example to LTPs, defensins, PR-10, storage proteins).

The level of knowledge about mango allergens is surely unsatisfactory, and further studies are required. Mango fruit certainly contain cross-reacting allergens, but the presence of species-specific allergens cannot be excluded.

\section{Abbreviations \\ SPT: skin prick test; CRD: component resolved diagnosis.}

\section{Authors' contributions}

NUS: study design, research material collection, interview with patient, obtained consent, prepared manuscript. EGU: evaluated and corrected the manuscript. MŻG: performed immunoassay. KL: performed immunoassay. $Ł S$ : evaluated and corrected the manuscript. ZB: evaluated the study design and the manuscript. All authors read and approved the final manuscript.

\section{Author details \\ ${ }^{1}$ Department and Clinic of Allergology, Clinical Immunology and Internal Diseases, Ludwik Rydygier Collegium Medicum in Bydgoszcz, NCU, Bydgoszcz, Poland. ${ }^{2}$ Division of Ergonomics and Exercise Physiology, Department of Hygiene, Epidemiology and Ergonomics, Ludwik Rydygier Collegium Medicum in Bydgoszcz, NCU, Bydgoszcz, Poland.}

Competing interests

The authors declare that they have no competing interests.

Availability of data and materials

The dataset supporting the conclusions of this article are included within the article.

Consent for publication

Patient consented to the publication of this case report. All authors consented to the publication

Funding

No funding source to declare. 


\section{Publisher's Note}

Springer Nature remains neutral with regard to jurisdictional claims in published maps and institutional affiliations.

Received: 7 May 2018 Accepted: 23 August 2018

Published online: 31 October 2018

\section{References}

1. Hegde VL, Venkatesh YP. Anaphylaxis following ingestion of mango fruit. J Invest Allergol Clin Immunol. 2007;17(5):341-4.

2. McGovern TW, LaWarre S. Botanical briefs: the mango tree-Mangifera indica L. Cutis. 2001;67:365-6.

3. Shah A, Gera K. Immediate hypersensitivity reaction with mango. Pneumonol Alergol Pol. 2014;82(5):445-53. https://doi.org/10.5603/ piap.2014.0058.

4. Sareen R, Shah A. Hypersensitivity manifestations to the fru-it mango. Asia Pac Allergy. 2011;1:43-9.

5. Himly M, Jahn-Schmid B, Dedic A, et al. Art v 1, the major allergen of mugwort pollen, is a modular glycoprotein with a defensin-like and a hydroxyproline-rich domain. FASEB J. 2003;17:106-8.

6. Tsai WC, Wu TC, Chiang BL, Wen HW. Cloning, expression, and purification of recombinant major mango allergen Man i 1 in Escherichia coli. Protein Expr Purif. 2017;130:35-43. https://doi.org/10.1016/j.pep.2016.06.009.

7. Paschke A, Kinder H, Zunker K. Characterization of cross-reac-ting allergens in mango fruit. Allergy. 2001;56:237-42.

8. van der Valk JPM, El Bouche R, van Wijk RG, de Groot H, Wichers HJ, Dubois AEJ, de Jong NW. Low percentage of clinically relevant pistachio nut and mango co-sensitisation in cashew nut sensitised children. Clin Transl Allergy. 2017;20(7):8. https://doi.org/10.1186/s13601-017-0145-z.

9. Gruber P, Gadermaier G, Bauer R, et al. Role of the polypeptide backbone and post-translational modifications in cross-reactivity of Art v 1, the major mugwort pollen allergen. Biol Chem. 2009:390:445-51.

10. Griffiths RLM, El-Shanawany T, Jolles SRA, Selwood C, Heaps AG, Carne EM, Williams PE. Comparison of the performance of skin prick, ImmunoCAP, and ISAC tests in the diagnosis of patients with allergy. Int Arch Allergy Immunol. 2017;172(4):215-23. https://doi. org/10.1159/000464326.
11. Bernardi ML, Giangrieco I, Camardella L, et al. Allergenic lipid transfer proteins from plant-derived foods do not immunologically and clinically behave homogeneously: the kiwifruit LTP as a model. PLOS ONE. 2011;6(11):e27856. https://doi.org/10.1371/journal.pone.0027856.

12. Ukleja-Sokołowska N, Gawrońska-Ukleja E, Żbikowska-Gotz M, Bartuzi Z, Sokołowski Ł. Sunflower seed allergy. Int J Immunopathol Pharmacol. 2016;29(3):498-503. https://doi.org/10.1177/0394632016651648.

13. Brehler R, Theissen U, Mohr C, Luger T. Latex-fruit syndrome: frequency of cross-reacting lgE antibodies. Allergy. 1997;52(4):404-10.

14. Paschke A, Kinder H, Zunker K, Wigotzki M, Steinhart H, Wessbecher $R$, Vieluf I. Characterization of cross-reacting allergens in mango fruit. Allergy. 2001;56(3):237-42.

15. Pablos I, Eichhorn S, Machado Y, Briza P, Neunkirchner A, Jahn-Schmid B, Wildner S, Soh WT, Ebner C, Park JW, PickI WF, Arora N, Vieths S, Ferreira F, Gadermaier G. Distinct epitope structures of defensin-like proteins linked to proline-rich regions give rise to differences in their allergenic activity. Allergy. 2018;73(2):431-41. https://doi.org/10.1111/all.13298.

16. Kahn IS. Fruit sensitivity. South Med J. 1942:35:858-9.

17. Silva R, Lopes C, Castro E, de Oliveira JF, Bartolomé B, Castel-Branco MG. Anaphylaxis to mango fruit and crossreactivity with Artemisia vulgaris pollen. J Invest Allergol Clin Immunol. 2009;19(5):420-2.

18. Ta V, Tsang RS, Avila EM, Leonard SA. Delay in diagnosis of severe food protein-induced enterocolitis syndrome caused by mango. Ann Allergy Asthma Immunol. 2015;115(2):157-8. https://doi.org/10.1016/j. anai.2015.05.022.

19. Weinstein S, Bassiri-Tehrani S, Cohen DE. Allergic contact dermatitis to mango flesh. Int J Dermatol. 2004;43(3):195-6.

20. Wiwanitkit V. Mango dermatitis. Indian J Dermatol. 2008;53(3):158. https //doi.org/10.4103/0019-5154.43215

21. Burks W. Skin manifestations of food allergy. Pediatrics. 2003;111(6 Pt 3):1617-24.

22. Boyce JA, Assa'ad A, Burks AW, Jones SM, Sampson HA, Wood RA, Plaut M, Cooper SF, Fenton MJ, Arshad SH, Bahna SL, Beck LA, Byrd-Bredbenner C, Camargo CA Jr, Eichenfield L, Furuta GT, Hanifin JM, Jones C, Kraft M, Levy BD, Lieberman P, Luccioli S, McCall KM, Schneider LC, Simon RA, Simons FE, Teach SJ, Yawn BP, Schwaninger JM. Guidelines for the diagnosis and management of food allergy in the United States: summary of the NIAIDsponsored expert panel report. Nutr Res. 2011;31(1):61-75. https://doi. org/10.1016/j.nutres.2011.01.001.
Ready to submit your research? Choose BMC and benefit from:

- fast, convenient online submission

- thorough peer review by experienced researchers in your field

- rapid publication on acceptance

- support for research data, including large and complex data types

- gold Open Access which fosters wider collaboration and increased citations

- maximum visibility for your research: over $100 \mathrm{M}$ website views per year

At BMC, research is always in progress.

Learn more biomedcentral.com/submissions 